\title{
Aprendiendo a Trabajar en Colaboración con \\ Mujeres de Bajos Ingresos Económicos en la Ciudad de Kingston, Ontario, Canadá: Reflexiones sobre un Proyecto de Investigación Acción Participativa
}

\author{
Pilar Camargo-Plazas ${ }^{1}$, Jennifer Waite ${ }^{1}$, Martha Whitfield ${ }^{1}$ y Lenora \\ Duhn ${ }^{1}$
}

\begin{abstract}
${ }^{1}$ School of Nursing, Queen's University, Ontario, Canada | mdpc@queensu.ca; jmw11@queensu.ca; m.whitfield@queensu.ca; duhnl@queensu.ca | https://orcid.org/00000002-8349-7723; https://orcid.org/0000-0002-7335-2176; https://orcid.org/0000-0002-76319056; http://orcid.org/0000-0002-0401-953X
\end{abstract}

\begin{abstract}
Resumen: El presente trabajo presenta los retos y desafíos generados por un proyecto de investigación acción participativa orientado a examinar el acceso a los servicios de salud y sociales para las mujeres que viven en condiciones de pobreza en Kingston, Ontario, Canadá. Objetivo: Describir nuestra experiencia implementando un trabajo participativo. Métodos: En este estudio usamos investigación acción participativa (IAP), investigación artística (foto voz), y metodologías interpretativas (hermenéutica fenomenológica). Resultados: Reportamos en este artículo nuestras experiencias con una organización sin animo de lucro en Kingston, así como también los desafíos y recompensas de este estudio. Igualmente, presentamos como hemos adaptado nuestro trabajo a las restricciones de COVID-19, mientras se sigue esforzando por mantener el impulso del estudio. Conclusión: Este proyecto nos ha dejado muchas enseñanzas como que requiere tiempo, adaptación y motivación para abordar una metodología participativa. Para lograr un cambio significativo y sostenido, debe habilitarse a través de una asociación, una que honre y beneficie a los más necesitados.
\end{abstract}

Palabras clave: Investigación Acción Participativa; Mujeres; Acceso; Sistema de Salud Servicios Sociales.

Learning to Work in Collaboration with Women Living on a Low-income in Kingston, Ontario, Canada: A Participatory Action Research Project

\begin{abstract}
This paper presents our experience following participatory methodologies to understand the experience of access to health and social services for women living on lowincome in Kingston, Ontario, Canada. Goals: The study aims are to describe and understand our experiences implementing a PAR study. Methods: In this study, we use participatory (PAR), art-based research (photovoice), and interpretive (hermeneutic phenomenological) approaches. Results: In this paper, we report our processes and experiences to date doing PAR with a not-for-profit organization in Kingston, Canada, both the challenges and the rewards, but most importantly the necessity of it for this context. This work has included the need to be adaptable and creative in light of COVID-19 restrictions, particularly while still striving to maintain study momentum. Conclusion: We have learned that a PAR study needs time, commitment and motivation. For meaningful, sustained change it must be enabled through partnership - one that honors and benefits those most in need
\end{abstract}

Keywords: Participatory Action Research; Women; Access; Health; Social Services.

\section{Introducción}

La proporción de canadienses que viven en hogares de bajos ingresos-definido como un hogar que recibe menos de la mitad de la renta mediana después de impuestos fue del 14.2\% (Statistics Canada, 2017a). Es decir que cerca de 1 en 7 o 4.9 millones de canadienses viven con bajos recursos económicos. La taza de pobreza para los hombres es de $13.4 \%$ y para las mujeres de $14.2 \%$ (Statistics Canada, 2017b). En la ciudad de Kingston con aproximadamente 123.798 habitantes el $14.8 \%$ vive en hogares de ingresos bajos. Siendo la taza de pobreza del $14 \%$ para los hombres y el $15.6 \%$ para las mujeres (Statistics Canada, 2017b). 
De acuerdo con esta información cerca de 19.000 mujeres viven en hogares de bajos ingresos en Kingston, Canadá, con las consecuencias que esto conlleva para la salud y bienestar físico y mental de este grupo poblacional. Se ha demostrado como aquellas mujeres que viven en hogares de bajos recursos enfrentan desigualdades que afectan su acceso integral a servicios sociales y de salud (Kiani et al., 2016; Socias et al., 2016). Todos estos problemas descritos demandan un proceso investigativo participativo.

La investigación acción participativa (IAP) ofrece el marco teórico y practico ideal para investigar las experiencias de acceso a los servicios de salud y sociales para las mujeres que viven con bajos recursos económicos en Kingston. Basado en el principio ético de la beneficencia un enfoque de acción participativa ayudará a estas mujeres, a obtener lo que es benéfico para ellas, o que promueva su bienestar y la justicia social (Braveman \& Gottlieb, 2014; Chan et al., 2018). El desarrollo de una IAP requiere reconocer los retos, desafíos y recompensas que conlleva el proceso colaborativo. Usando como ejemplo el proyecto con mujeres de Kingston presentamos en este artículo nuestras experiencias, reflexiones, adaptaciones y modificaciones que hemos hecho de este proyecto a través del tiempo. A la luz de los siguientes cuestionamientos asumimos la tarea de compartir nuestra experiencia con la IAP. Entonces a través del texto presentamos ¿cuáles son los retos, desafíos y recompensas de la IAP?, ¿qué inspira nuestro trabajo?, ¿qué hemos aprendido al embarcarnos en un proyecto IAP? A continuación, presentamos una descripción de nuestro estudio, las metodologías y métodos utilizadas en este estudio, las respuestas a las preguntas formuladas arriba, finalizando con una descripción de nuestro aprendizaje y conclusión.

\section{El Estudio}

Siguiendo los lineamientos de una IAP, investigación artística y metodologías interpretativas, y en colaboración con un grupo de mujeres examinaremos como el género y el estatus socioeconómico afectan el acceso a los servicios sociales y de salud para las mujeres. Este trabajo de investigación ofrece la oportunidad para que mujeres, investigadores, profesionales de la salud y tomadores de decisiones determinen la forma más efectiva de atender a las necesidades de acceso a los servicios de salud y social identificados por los participantes de la investigación. El proyecto está desarrollado en colaboración con la organización sin ánimo de lucro Saint Vincent de Paul (SVDP) quien se encarga de brindar apoyo a personas y familias de bajos recursos económicos en Kingston.

Esta investigación fue aprobada por el comité de ética de Queen's University, en Kingston, Ontario. Tenemos planeado invitar a 20 mujeres de la comunidad con edades entre los 20-75 años. Las actividades de investigación incluyen foto voz, entrevistas individuales, círculos culturales, y movilización de resultados. Estas actividades serán explicadas en las siguientes secciones. El análisis de datos es participativo y sigue un lineamiento temático (van Manen, 2014). Para el análisis de datos seguimos el marco teórico de los determinantes sociales de la salud descritos por Loppie-Reading and Wien (2013) quienes clasifican los determinantes en: (1) distales o aquellos determinantes que a nivel macro afectan la salud de una población (ej. Aspectos estructurales, políticos, económicos y sociales de una nación); (2) intermedios son el origen de los determinantes proximales (ej. La infraestructura de una comunidad, sus recursos, sistemas y capacidades); y (3) proximales o aquellos determinantes que impactan la salud física, emocional, espiritual y mental de una población (ej. Hábitos de vida saludable, medio ambiente, educación, estatus socioeconómico). Este marco teórico guiará el análisis de datos y nos ayudará crear temas que serán abordados en los círculos culturales. En las siguientes secciones se presenta una descripción breve de las metodologías y métodos de recolección de datos, así como los desafíos y lecciones aprendidas hasta el momento en el desarrollo de nuestro proyecto participativo. 


\section{Metodologías y Métodos}

La IAP ofrece un enfoque participativo y de aprendizaje colectivo transformador y orientado al cambio social (Hacker, 2017; Oetzel et al., 2018). Miembros de la comunidad, representantes de organizaciones y los investigadores trabajan unidos en la coproducción de conocimiento que es práctico, colaborativo y potenciador (Hacker, 2017; Oetzel et al., 2018; Springer \& Skolarus, 2019). En nuestro estudio empleamos un marco participativo para explorar como la distribución de los determinantes sociales (ej. Estatus socioeconómico y género) contribuyen a las desigualdades sociales y de salud de mujeres que viven en Kingston.

La investigación artística es una metodología cualitativa que utiliza procesos y expresiones artísticas como fuente primaria para explorar, comprender y representar la experiencia humana (Segal-Engelchin et al., 2020; Wang et al., 2017). La esencia de la investigación artística es el de crear colaboraciones entre miembros de la comunidad, participantes e investigadores los cuales coproducen un conocimiento único (Ottewell-Watson, 2014; Segal-Engelchin et al., 2020; Wang et al., 2017). En nuestro estudio, la investigación artística y especialmente la foto voz nos ayudará a explorar en profundidad las experiencias de acceso a los servicios sociales y de salud para las mujeres que viven en Kingston.

Igualmente, nuestro estudio sigue un enfoque fenomenológico hermenéutico para el análisis de datos (van Manen, 2014). Este método explora diferentes temas existenciales dimensiones humanas como la corporalidad, la relacionalidad, la temporalidad y la espacialidad (van Manen, 2014). Hasta la fecha, en nuestros trabajos de investigación, este enfoque ha sido capaz de capturar las experiencias vividas por grupos vulnerables a través de relatos escritos que representan su vida de manera profunda (Camargo Plazas et al., 2012; Cameron et al., 2014).

En nuestro estudio planeamos usar una combinación de métodos para describir y entender las experiencias de vida de las mujeres en Kingston, incluyendo (foto documentación, foto texto y entrevistas individuales), círculos culturales (Freire, 2013) y exhibición de arte como método de movilización de resultados. El objetivo es proporcionar varios niveles de reflexión a los participantes acerca de sus experiencias personales, así como ir más allá de nuestra agenda de investigación para facilitar la cooperación de los participantes como investigadores del proyecto (Liebenberg, 2018). Foto voz será usada para descubrir esas experiencias difíciles de capturar con palabras. Como describen Wang and Burris (1997), la foto voz es un método de recolección de datos que incorpora las voces de los participantes mediante el uso de imágenes (Evans-Agnew \& Rosemberg, 2016; Wang \& Burris, 1997). Nuestro análisis de datos seguirá el método fenomenológico hermenéutico descrito por van Manen (2014) utilizando el marco teórico de los determinantes sociales de la salud descritos por Loppie-Reading y Wien (2013).

Finalizado el análisis de datos invitaremos a las mujeres a participar en un círculo cultural (Freire, 2013). El objetivo de los círculos de cultura es la concientización (Freire, 2000, 2013). En nuestro estudio planeamos abrir esos espacios para el diálogo donde rompamos con esa cultura de silencio que viven las mujeres de bajos ingresos económicos en Kingston. A través de los círculos de cultura, investigadores y mujeres, nos reuniremos para discutir la realidad local y nacional del acceso a los servicios sociales y de salud para las canadienses, creando un espacio donde todos aprendamos a analizar y crear estrategias de cambio que mejoren la realidad de vida de las mujeres de esta comunidad. Cuando las restricciones por la pandemia lo permitan planeamos organizar una exhibición de las fotos dentro de la comunidad para así identificar los problemas y crear soluciones dentro de la comunidad de Kingston. En la siguiente sección presentamos una descripción de nuestra experiencia con el proyecto desde su financiación hasta el día de hoy. 


\subsection{Desarrollo de la Colaboración}

En enero del 2020 iniciamos conversaciones con la directora de SVDP Judy Fife, a quien presentamos nuestro proyecto. Durante nuestro primer encuentro, Judy expreso entusiasmo a nuestra idea de involucrar a mujeres de la comunidad para explorar sus experiencias de acceso a los servicios de salud y sociales. El proceso participativo requirió varios encuentros en los cuales dos investigadoras visitaron el lugar durante tres semanas y entablaron conversaciones informales con mujeres usuarias de los servicios de SVDP. En la próxima sección relataremos de forma más detallada estas experiencias.

\subsection{Comité Asesor}

La formación de un comité asesor para nuestro proyecto ha sido fundamental para supervisar el proceso de investigación. El comité asesor consta de tres miembros de SVDP, una enfermera de salud pública, una voluntaria de foto voz un centro comunitario local y una abogada que trabaja en el área de justicia social. En septiembre de 2020 fue nuestra primera reunión para organizar nuestras actividades de investigación. Una de nuestras metas es involucrar en el comité asesor a una mujer de la comunidad. Una de nuestras metas es involucrar en el comité asesor a una mujer miembro de la comunidad. Lo cual está a punto de concretarse con la ayuda de Judy. Esta persona traerá al comité la perspectiva de las mujeres que viven en Kingston.

\section{Retos, Desafíos y Recompensas}

La naturaleza interactiva del trabajo participativo se enfoca en el desarrollo de alianzas entre los investigadores y miembros de la comunidad (Coughlin et al., 2017). El rol de los comités asesores en IAP es el de aportar la perspectiva interna de la comunidad al proceso de la investigación. Al desarrollar este estudio participativo no anticipamos muchas situaciones que nos han hecho cambiar de dirección, afortunadamente fortaleciendo nuestro trabajo de investigación. Por ejemplo, inicialmente el proyecto estaba enfocado a investigar las experiencias de acceso a los servicios de salud y sociales para las mujeres inmigrantes (ej. Residentes legales, refugiadas e inmigrantes indocumentadas) en Canadá. Trabajo que planeamos con un centro comunitario en Ottawa. Sin embargo, un cambio en la administración y manejo del centro comunitario nos llevó a buscar otro sitio para el desarrollo de nuestra investigación. Inmediatamente, pensamos en Toronto, una ciudad multicultural. Allí nos conectamos con dos organizaciones que inicialmente estaban interesadas en el estudio. Sin embargo, al presentarles la descripción de las actividades en su versión original sin ser modificadas cada uno de ellos decidió suspender su participación. Creemos que una estrategia fundamental es el lograr confianza, mantenerla y materializarla a través del dialogo con las comunidades. La mayoría de las veces el trabajo financiado es diferente del trabajo que se realiza con la comunidad. Entonces proyectos IAP deben ser modificados de acuerdo con las necesidades de la comunidad.

Otro desafío que no consideramos fue la ubicación de Kingston a tres horas de Toronto. Estar lejos de la comunidad y los viajes constantes se convirtieron en un problema para interconectar con comunidades de mujeres en Toronto. Por esta razón decidimos replantear el proyecto con un grupo de mujeres a nivel local. Como mencionamos al inicio de este artículo, la ciudad de Kingston es una ciudad intermedia de contrastes, con áreas donde viven personas y familias con altos ingresos económicos y sitios en el centro y áreas rurales de pobreza extrema (Statistics Canada, 2017b). Aprendimos como IAP requiere una conexión y dialogo continuo con miembros de la comunidad por esta razón creemos que las colaboraciones deben iniciarse a nivel local para así crear vínculos y relaciones mucho más fuertes y duraderas. Siempre se debe iniciar a nivel local y luego expandir el trabajo a otras regiones. 
Una vez decidido el nuevo rumbo del proyecto buscamos agencias en Kingston que estuvieran interesadas en este proyecto. SVDP fue ese sitio. En enero y febrero del 2020, asistimos a SVDP todas las mañanas de los miércoles y jueves para interactuar con miembros de la comunidad y hablarles a las mujeres acerca de nuestro proyecto. La verdad más que una estrategia de reclutamiento de participantes queríamos crear vínculos y que la comunidad viera que no pretendíamos solamente colectar información y abandonarlos. Vivimos encuentros muy significativos con los voluntarios, hombres y mujeres miembros de la comunidad. En conversaciones informales con mujeres que asisten a SVDP aprendimos acerca de la realidad de pobreza en la que muchas mujeres viven. En alguna oportunidad una mujer comentó a una de las investigadoras como por la falta de vivienda estable no podía acceder a los servicios de salud para su enfermedad crónica. Lograr la confianza y mantenerla ha sido posible a través del diálogo permitiendo acercamientos y la posibilidad de planear y organizar el trabajo de una manera más efectiva. De la mano de los miembros de nuestro comité asesor hemos podido delinear un trabajo enfocado en las necesidades de las mujeres de Kingston.

En marzo del 2020, la pandemia por COVID-19 se declaró a nivel mundial y nuestro trabajo fue nuevamente detenido por la cuarentena y otras medidas de protección de la salud. Nuevamente con la ayuda de nuestro comité asesor hemos logrado adaptar nuestro proyecto a la pandemia. En conversación con la directora de SVDP y nuestro comité asesor hemos creado un paso a paso de actividades que permitan la protección de la salud de las participantes y el desarrollo de nuestras actividades siguiendo los lineamientos y guías de interacción durante COVID-19. Una vez las restricciones se han levantadas, y con el ideal de salvaguardar y proteger a los miembros de esta comunidad iniciaremos en las semanas que vienen nuestras actividades de investigación. Hasta la fecha hemos reclutado diez participantes, creemos que el proyecto, aunque lento ha logrado interesar a las mujeres quienes encuentran en la foto voz una forma de expresar sus experiencias de vida.

\section{Conclusiones}

Nuestro trabajo sigue evolucionando y aunque anticipamos más aprendizaje en el futuro, hay algunas observaciones claves que hemos hecho sobre el proceso. Como en cualquier relación, trabajar con miembros de la comunidad requiere de tiempo para crear lazos de confianza y muto respeto. Es recordar constantemente la perspectiva participativa del proyecto, lo que implica el beneficiar a un grupo de mujeres creando con ellas soluciones a sus problemas de salud y bienestar social. La IAP se transforma en un proceso relacional, de aprendizaje colectivo y democrático que constantemente nos recuerda como nuestros pensamientos y acciones deben basarse en lo que es correcto para las mujeres involucradas en este estudio, no en lo que podría ser más fácil para los investigadores, y eso requiere observar, hacer preguntas y escuchar activamente a las necesidades del otro. Deben ser sus voces, no las nuestras, las que se escuchen; solo así habremos permanecido fieles al principio de beneficencia. Por último, la actual situación a nivel mundial de la pandemia nos ha enseñado a ser pacientes y adaptarnos a los cambios inesperados sin dejar de ser creativas.

\section{Agradecimientos}

Queremos agradecer el apoyo desinteresado de la Señora Judy Fyfe, Directora Ejecutiva de St. Vincent de Paul Society of Kingston, Ontario. Igualmente agradecemos a los miembros de nuestro comité asesor. Finalmente agradecemos a Social Science and Humanities Research Council particularmente al programa Insigh Development Grants (SSHRC-IDG) por el financiamiento de este proyecto. 


\section{Referencias}

Braveman, P., \& Gottlieb, L. (2014, Jan-Feb). The social determinants of health: it's time to consider the causes of the causes. Public Health Rep, 129 Suppl 2, 19-31. https://doi.org/10.1177/00333549141291S206

Camargo Plazas, M. d. P., Cameron, B. L., \& Smith, D. G. (2012, Apr-Jun). Neoliberal-oriented health care system answer to global competition or a threat to health equality for people with chronic illness. ANS Adv Nurs Sci, 35(2), 166-181. https://doi.org/10.1097/ANS.0b013e31825372a4

Cameron, B. L., Carmargo Plazas Mdel, P., Salas, A. S., Bourque Bearskin, R. L., \& Hungler, K. (2014, Jul-Sep). Understanding inequalities in access to health care services for aboriginal people: a call for nursing action. ANS Adv Nurs Sci, 37(3), E1-E16. https://doi.org/10.1097/ANS.0000000000000039

Chan, C. W. T., Gogovor, A., Valois, M. F., \& Ahmed, S. (2018, Jun 19). Age, gender, and current living status were associated with perceived access to treatment among Canadians using a cross sectional survey. BMC Health Serv Res, 18(1), 471. https://doi.org/10.1186/s12913018-3215-6

Coughlin, S., Smith, S., \& Fernández, M. (2017). Handbook of community-based participatory research. Oxford University Press.

Evans-Agnew, R. A., \& Rosemberg, M. A. S. (2016). Questioning photovoice research: Whose voice? . Qualitative Health Research, 26(8), 1019-1030. https://doi.org/https://doi.org/10.1177/1049732315624223

Freire, P. (2000). Pedagogy of the oppressed (30th Anniversary ed ed.). Bloomsbury.

Freire, P. (2013). Education for critical consciousness. Bloomsbury Academic.

Hacker, K. (2017). Community-based participatory research. SAGE Publications Ltd.

Kiani, Z., Simbar, M., Dolatian, M., \& Zayeri, F. (2016, Sep 1). Correlation between Social Determinants of Health and Women's Empowerment in Reproductive Decision-Making among Iranian Women. Glob J Health Sci, 8(9), 54913. https://doi.org/10.5539/gjhs.v8n9p312

Liebenberg, L. (2018). Thinking critically about photovoice: Achieving empowerment and social change. International Journal of Qualitative Methods, 17, 1-9. https://doi.org/https://doi.org/10.1177/1609406918757631

Loppie-Reading, C., \& Wien, F. (2013). Health inequalities and social determinants of Aboriginal people's health. National Collaborating Centre for Aboriginal Health (NCCAH). https://www.nccih.ca/docs/determinants/RPT-Healthlnequalities-Reading-Wien-EN.pdf

Oetzel, J., Wallerstein, N., Duran, B., Sanchez-Youngman, S., Nguyen, T., Woo, K., Wang, J., Schulz, A., Keawe'aimoku Kaholokula, J., Israel, B., \& Alegria, M. (2018). Impact of participatory health research: A test of the Community-Based Participatory Research Conceptual Model. BioMed Research International, 7281405-7281412. https://doi.org/https://doi.org/10.1155/2018/7281405

Ottewell-Watson, S. (2014). De-mythicizing art-based research: An art therapy student's dance with a creative methodology (Démystifier la recherche par l'art: Une étudiante en art thérapie utilise une méthodologie créative par la danse). Canadian Art Therapy Association Journal, 27(2), 9-12. https://doi.org/https://doi.org/10.1080/08322473.2014.11415595

Segal-Engelchin, D., Huss, E., \& Massry, N. (2020). Arts-based methodology for knowledge coproduction in social work. The British Journal of Social Work, 50(4), 1277-1294. https://doi.org/https://doi.org/10.1093/bjsw/bcz098

Socias, M. E., Koehoorn, M., \& Shoveller, J. (2016, Jan-Feb). Gender Inequalities in Access to Health Care among Adults Living in British Columbia, Canada. Womens Health Issues, 26(1), 74-79. https://doi.org/10.1016/j.whi.2015.08.001

Springer, M., \& Skolarus, L. (2019). Community-based participatory research: Partnering with communities. Stroke, 50(3), e48-e50. https://doi.org/https://doi.org/10.1161/STROKEAHA.118.024241 
Vol. 8 | Investigación Cualitativa en Salud: Avances y Desafíos

Statistics, Canada,.. (2017a). Focus on Geography Series, 2016 Census. Statistics Canada Catalogue no. 98-404-X2016001. Data products, 2016 Census.

https://www12.statcan.gc.ca/census-recensement/2016/as-sa/fogs-spg/Facts-cmaeng.cfm? $\mathrm{LANG}=\mathrm{Eng} \& \mathrm{GK}=\mathrm{CMA} \& \mathrm{GC}=521 \& \mathrm{TOPIC}=1$

Statistics, Canada,.. (2017b). Kingston, CY [Census subdivision], Ontario and Canada [Country] (table). Census Profile. 2016 Census. Statistics Canada Catalogue no. 98-316-X2016001. https://www12.statcan.gc.ca/census-recensement/2016/dp-pd/prof/index.cfm?Lang=E

Van Manen, M. (2014). Phenomenology of practice. Meaning-giving methods in phenomenological research and writing. Left Coast Press.

Wang, C., \& Burris, M. A. (1997). Photovoice: Concept, methodology, and use for participatory needs assessment. Health Education \& Behavior, 24(3), 369-387. https://doi.org/https://doi.org/10.1177/109019819702400309

Wang, Q., Coemans, S., Siegesmund, R., \& Hannes, K. (2017). Arts-based methods in socially engaged research practice: A classification framework. Art/Research International: $A$ Transdisciplinary Journal, 2(2), 5-39. https://doi.org/https://doi.org/10.18432/R26G8P 